\title{
Preliminary design of an alternative energy storage system for a city car based on flywheel
}

\author{
Michele Vignati ${ }^{1}$, Davide Tarsitano, Francesco Braghin and Federico \\ Cheli \\ Politecnico di Milano \\ Department of Mechanical Engineering \\ via La Masa 1, 20156, Milan, Italy
}

\begin{abstract}
Vehicle energy storage systems have a crucial impact on the spreading of hybrid and electric vehicles. Nowadays the most adopted solution is represented by batteries. This solution suffers of aging phenomenon that reduces the battery capacity; moreover, the battery recycling at the end of its life is still a challenge and generate pollution. This paper presents a preliminary design of a kinetic energy storage system intended for city micro-car. The energy is stored by means of high rotating flywheel. First, an energetic model of the car powertrain including flywheel and bearings is proposed and used to evaluate the car energy requirement to accomplish its typical journey. Then, material, geometry and motor have been selected to fit all the vehicle space requirements considering also that flywheels operate in vacuum environment. The paper than presents an analysis on the gyroscopic effects induced by the flywheel when it is subjected to car chassis acceleration in typical urban driving scenario. Finally, preliminary considerations on bearing layout are drawn by comparing different bearings types. Bearing loads have been evaluated with a multibody model that is fed with measured car chassis acceleration data.
\end{abstract}

keywords: flywheel, kinetic energy storage system, electric vehicles

\section{Biographical notes:}

Michele Vignati received his master degree (2013) and PhD (2017) in mechanical engineering in Mechanical Department of Politecnico di Milano with thesis on control strategies for distributed powertrain of hybrid and electric vehicles. From 2019 he is Assistant Professor in the research field of applied mechanics. In particular, he focuses on mechanical systems

\footnotetext{
${ }^{1}$ corresponding author: michele.vignati@polimi.it
} 
dynamics and control applied in the automotive field. He worked on tire dynamics and modelling in cooperation with Pirelli while in the last years he is also working in autonomous driving field.

Davide Tarsitano graduated from the Politecnico di Milano in 2005, he obtained the title of PhD in Mechanical Systems Engineering in 2010 with a numerical-experimental research thesis entitled: "Development of an innovative prototype of Plug-In hybrid vehicle: power train control strategies for improving energy efficiency.". From 1 June 2010 he took up a position as Researcher at the Mechanics Department of the Politecnico di Milano. Since 2019 he is Associate Professor in the same department. The research activity mainly concerns the field of mechatronics with particular reference to controlled electric drives and their use on mechanical systems. He is currently involved in numerous research projects and contracts relating to the study of hybrid and electric vehicles.

Francesco Braghin Graduated from the Politecnico di Milano in 1997, he obtained the title of PhD in Applied Mechanics in 2001. Since October 1999 he has been recipient of a research grant for the research program called "Wheel-rail interaction" at the Department of Mechanics of the Politecnico di Milano. Since 1 May 2001 he has been working as a Researcher at the Department of Mechanics of the Politecnico di Milano. In November 2010 he was called as Associate Professor at the Faculty of Industrial Engineering of the Politecnico di Milano and since 2015 he is Full Professor in Mechanics Applied to Machines at the Politecnico di Milano. Author of about 250 scientific publications and responsible for several research projects / contracts, his most recent research activity is in the field of vehicle dynamics (both road and rail) and mechatronics. In particular, as regards vehicles, it deals with tire modeling and their interaction with the ground, as well as with the application of excellent control algorithms to the design of hybrid and electric vehicles. As regards mechatronics, the research focuses on the development of integrated solutions in different application areas as well as on the development and use of passive and active metamaterials for vibration isolation.

Federico Cheli graduated in Mechanical Engineering in 1981 at the Milan Polytechnic. In 1983 he was appointed university researcher, in 1992 a second-level university professor and since 2000 he is full professor at the Faculty of Industrial Engineering of Politecnico di Milano. The scientific activity mainly concerned research on dynamic behavior of vehicles, road and rail, as weel as large structures subjected to wind and traffic; identification of the parameters of mechanical systems and their control; modeling and testing of the tire and its interaction with the vehicle; studies on the aerodynamics of rail and road vehicles; studies and design of hybrid / electric vehicles. He is currently chairman of the board of the mechanical engineering course of the faculty of industrial engineering of the Politecnico di Milano; he is a member of the University Didactic Coordination 
Body. He is part of the Board of the Applied Mechanics Group and of the kinematics and dynamics group of the multibody systems of the Italian Association of Theoretical and Applied Mechanics. He is coordinator of numerous national research projects (PRIN) and Europe and responsible for a series of research contracts between the Politecnico di Milano and national and international companies. Member of the editorial board of the International Journal of Vehicle Performance and of the International Journal of Vehicle Systems Modeling and Testing.

\section{INTRODUCTION}

In recent years environment care and pollution reduction pushes the increase of fuels quality and more adequate after-treatment systems, anyway emissions are still present. Electrical motors and batteries seem a promising option to solve this issue and almost every car manufacturer has created its own prototypes.

The main drawback of batteries is low energy density (one order of magnitude lower than gasoline) that directly leads to heavy and low driving-range cars. Moreover, battery performance decay in time [5] due to aging phenomenon, their recycling and production processes produce pollutant emission. Full electric vehicles are widespread, but for car applications the presence of an internal combustion engine is still dominant as most of electrified vehicles present hybrid solutions instead of full electric ones. In hybrid vehicle the battery is smaller and driving range remains comparable with fossil fuel vehicles [25, 22].

Few alternative solutions for storing energy are present, one is represented by flywheels [21]. Flywheels store energy by means of kinetic energy, which depends on body inertia and the square power of rotating speed. The flywheelkinetic energy is than transformed into electric energy to power the traction electric motor thus decelerating the flywheel. The process is reversible, when the vehicle brakes, energy is recovered and stored into the flywheel by increasing its speed. As for batteries, several power-train configurations are possible and a correct layout of the power flow in the vehicle must be designed. These solutions are also known under the name Flywheel Energy Storage System (FESS). One of the very first applications of flywheel as an energy storage system for vehicles was the Gyrobus $([9,13])$ presented in 1950 that used a flywheel $(1500$ $\mathrm{kg}, 1.6 \mathrm{~m}$ diameter) as unique source of energy and a Continuously Variable Transmission (CVT). This system suffers of low driving-range which causes its decommissioning in 1969.

As per batteries, the obtainable driving range is the main problem since the weight of the flywheel increases the energy request to drive the car. Several form of hybrid powertrain layouts tried to partially solve the problem [18, 19]. As shown in [9], both series and parallel hybrid configuration are available. Series configuration is obtained by positioning the flywheel downstream the gearbox. Internal Combustion Engine ICE-flywheel and flywheel-wheels connections are CVTs, since this type of transmission guarantees the optimal rotating speed for each component. Although, having a small transmission ratio limits the 
maximum speed of the flywheel. This results in bigger dimensions (high specific moment of inertia) and weight (higher mass) to have a sufficient energy storage. This creates room and safety problems. On the other hand, parallel configuration $[18,25]$ offers several possibilities mainly depending on where the flywheel is placed into the power-chain. During the last decade, however, this technology has become so mature that some of the major car manufacturers have succeeded in installing it on series models ([16, 20, 4, 23]).

Many authors in literature faced different problems related to flywheels.

Imer and others [28] studied the flywheel mechanical resistance due to geometry induced stresses. In [7] the problem associated with the adoption of magnetic bearings is faced. In this paper also a choice of the flywheel material is reported based on stress analysis. Conteh in [6] deeply concentrates on the design of laminate composite material for flywheel application.

Ahrens in Ahrens1996 studied different control strategy for active magnetic bearings for static storage system adopting flywheel. Kubo in Kubo2003BasicDO presents a preliminary design for a $1 \mathrm{kWh}$ flywheel storage system for static applications. Rozewicz in [24] presents instead a geometry optimization of a flywheel for increasing the energy density of the system while satisfying maximum stress constraints for simple flywheel shape.

Considering the flywheel characteristics, flywheel technology seems to be a possible energy storage solution for short range urban application while for long range hybrid solutions must be adopted. Moreover, flywheel is competitive with batteries when high currents are required in typical driving transients maneuvers where both charging and discharging can be easily supported by flywheels. For this reason, their utility in the transport field is best expressed during accelerations and deceleration, typically on urban routes. This work tries to go beyond studying the possibility to install a flywheel energy storage system on a small city car.

This paper presents a systemic point of view that combining the aspects presented above produces the preliminary design of a flywheel based storage system to power a urban quadricyle.

The design accounts for the energetic model of the system which is necessary to evaluate the required energy of the storage system. The energetic model is used iteratively to obtain the flywheel energy since the flywheel energy depends also on it mass which is then affecting the power consumption of the car. The proposed method is repeated with different flywheel material accounting for constraints given by maximum flywheel size to fit into the target car, and by accounting for material limit stress for a simplified flywheel geometry. Once the flywheel size is determined, the bearings configuration is analyzed considering different bearing types. Loads on the bearings are evaluated by means of multibody simulations where the car chassis motion is taken from real measured data in urban scenario.

The paper is thus organized as follows. Section 2 illustrates the application case, the model that is used for energy consumption simulations proposing a powertrain layout that includes the flywheel. Section 4 explained the procedure used to calculate the necessary flywheel mass to fulfill the requirements 
Table 1: Car main data

\begin{tabular}{lcr}
\hline Parameter & unit & value \\
\hline seats & - & 2 \\
length & {$[\mathrm{mm}]$} & 2765 \\
width & {$[\mathrm{mm}]$} & 1540 \\
height & {$[\mathrm{mm}]$} & 1525 \\
wheelbase & {$[\mathrm{mm}]$} & 1765 \\
ride height & {$[\mathrm{mm}]$} & 150 \\
weight without battery pack & $\mathrm{kg}$ & 398 \\
transportable weight & $\mathrm{kg}$ & 200 \\
driving-range & $\mathrm{km}$ & 120 \\
front suspension & & McPherson \\
rear suspension & & Watt's linkage \\
front tires & & $145 / 60 \mathrm{R} 13$ \\
rear tires & & $165 / 55 \mathrm{R} 13$ \\
motor maximum torque & {$[\mathrm{Nm}]$} & 20 \\
motor nominal power & {$[\mathrm{kW}]$} & 6 \\
motor maximum speed & {$[\mathrm{rpm}]$} & 3500 \\
motor final drive ratio & & 4.08 \\
battery voltage & $\mathrm{V}$ & 72 \\
battery energy & $\mathrm{kWh}$ & 10.8 \\
\hline
\end{tabular}

of the driving cycle. In section 5 attention is given on dynamic effects arising when coupling the flywheels with the vehicle. Gyroscopic effects are evaluated together with bearing loads. Lastly a possible bearing configuration is shown in section 6 .

\section{Case study}

Before going into details of the flywheel design, it is necessary to investigate the car energy requirement and the rough dimension of the flywheel in terms of weight and required space. The considered car is an urban quadricycle whose main data are reported in Table 1 (see also [26]). The flywheel has to replace high voltage battery pack, preserving the other components of the power-train.

To simplify the design process in this initial stage, the following hypothesis are considered:

1. the flywheel has a rim geometry with constant thickness since this has a higher specific moment of inertia with respect to disk shape. The radial dimension is constrained to fit the flywheel into the selected car.

2. the number of flywheels is fixed to two for two reasons:

- flywheel must be an even number with opposite spin to balance gyroscopic effects; 


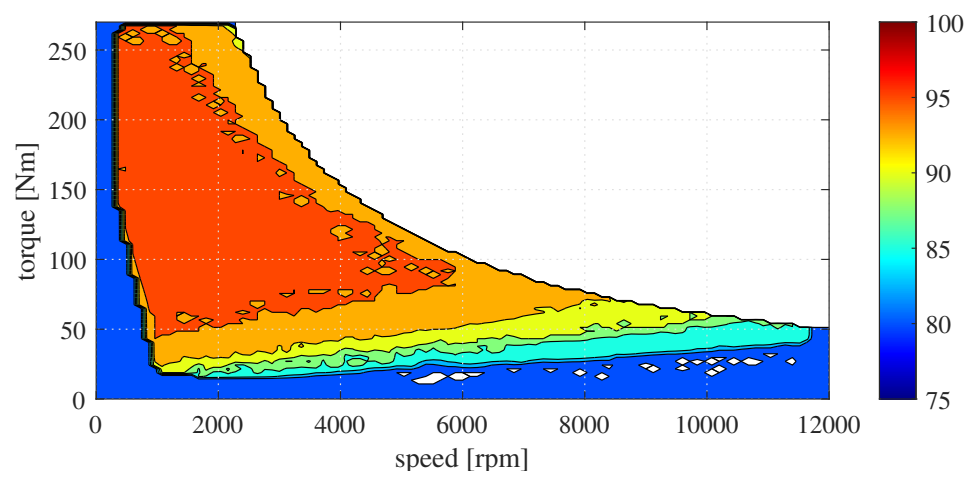

Figure 1: Motor 1 efficiency map

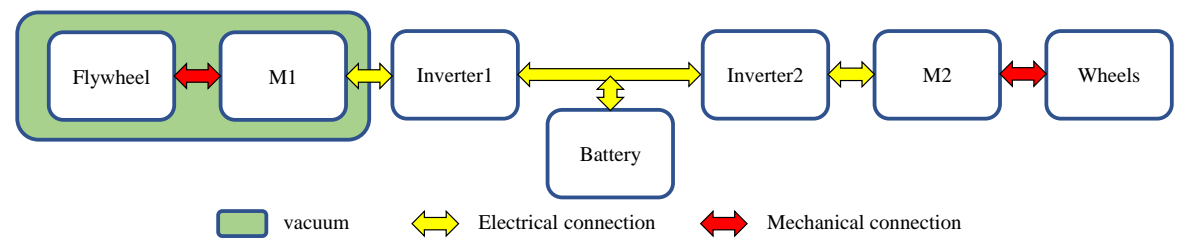

Figure 2: fig.PowertrainLayout

- given the small size of the car, two is the maximum number of flywheel that can be stored on board the vehicle.

3. a vacuum environment for the flywheel is essential to guarantee limited air friction losses.

Given those assumptions, the power-train layout of the car has been modified as shown in figure 2 . The energy source, the flywheels, are electrically connected to the wheels by means of electric motors. M1 is mechanically connected to the flywheel while M2 is connected to the wheels. The two motors work at the same common DC bus voltage while the nominal speed is significantly different. To correctly represent the available power, the motor efficiency map (see Figure 1) is used to evaluate the maximum motor torque and efficiency.

A small battery pack ( $500 \mathrm{Wh}$ ) is still present since it acts as a buffer between the two motor inverters and it smooths the currents profile. For this reason, a suitable logic is necessary to handle the power flow from and to the battery and the flywheel. The authors decide to power and discharge the battery before charging and discharging the flywheel, i.e. when the vehicle requires energy, firstly the battery is discharged down to the $40 \%$ of its capacity then the flywheel is discharged. When the car brakes first the battery is recharged and only when this is fully charged, the remaining energy is stored into the flywheel.

Given those assumptions, the energy request from the car must be evaluated to size the flywheels. Considering the category of the selected car, whose average 


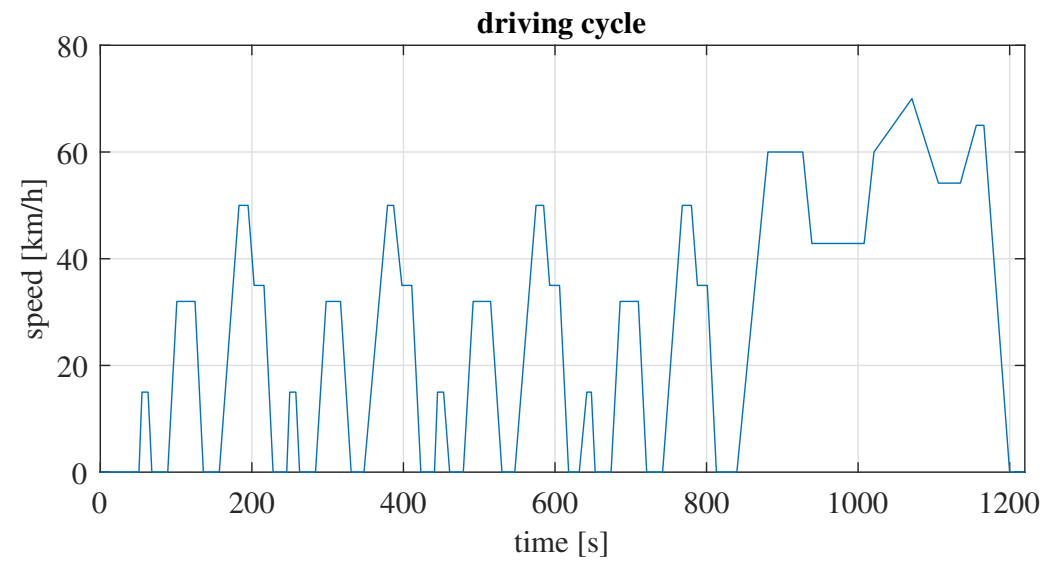

Figure 3: Modified NEDC cycle. The cycle is repeated to cover a total length of $50 \mathrm{~km}$.

mission length of about $50 \mathrm{~km}$ since it is typically used in an urban context [14], the speed profile shown in Figure 3 has been adopted. It is a modification of the classical NEDC cycle that considers slower speed typical of the selected vehicle.

The model considers the vehicle running at full load with two people on board. Rolling and aerodynamic resistances are considered, as well as the power consumption due to the auxiliaries. Power losses of the two motors (plus inverter) are considered through efficiency maps.

\section{$3 \quad$ Vehicle energetic model}

The model equations adopted to simulate the energy request while driving are here reported. Adopting a point mass representation of the vehicle, the mechanical power $\Pi_{m}$ required to move and accelerate the car is

$$
\Pi_{m}=\left(m_{e q} \dot{v}+F_{\text {aero }}+F_{\text {rol }}\right) v
$$

where $v$ is the car speed, $m_{e q}$ is the total equivalent inertia of the car accounting for car mass, flywheel mass and rotating inertia. $F_{a e r o}$ is the aerodynamic resistance force

$$
F_{\text {aero }}=\frac{1}{2} b v^{2}
$$

where $b$ is a constant that accounts for drag coefficient, frontal surface, air density. $F_{\text {rol }}$ the rolling resistance defined as

$$
F_{\text {rol }}=f_{v} m g
$$

where $f_{v}$ is the rolling resistance coefficient, $m$ is the total vehicle mass and $g$ is gravity acceleration. 


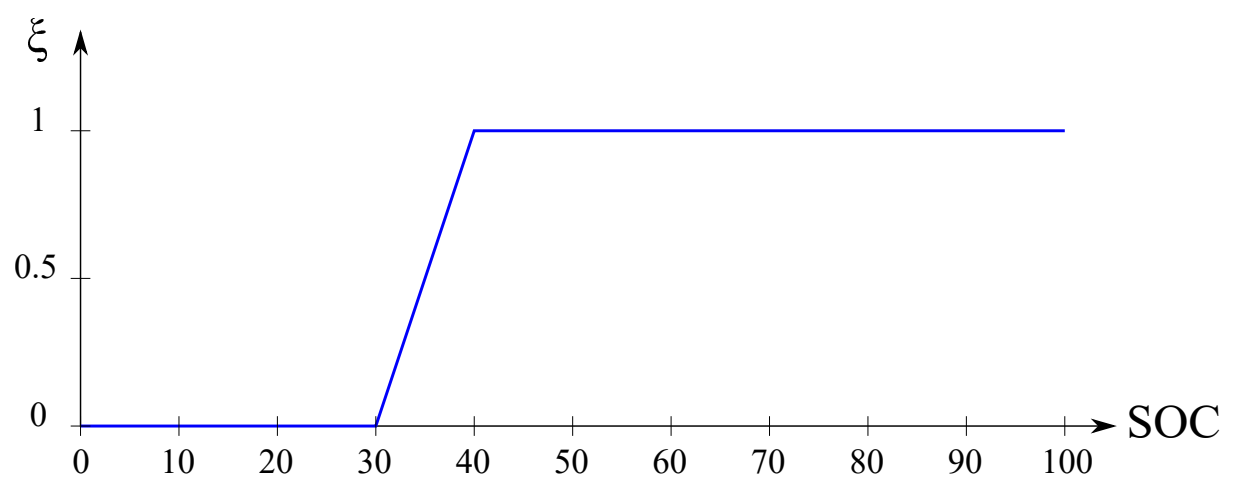

Figure 4: Flywheel discharge factor based on battery SOC.

The electric power required to move the car is derived from mechanical power by considering the driving motor plus inverter efficiency

$$
\Pi_{e 2}=\eta_{i 2} \eta_{m 2}\left(T_{m}, \omega_{m}\right) \Pi_{m}
$$

where the inverter efficiency $\left(\eta_{i 2}\right)$ is assumed as constant while the motor efficiency $\left(\eta_{m 2}\left(T_{m}, \omega_{m}\right)\right)$ is a function of motor torque $\left(T_{m}\right)$ and speed $\left(\omega_{m}\right)$ through the motor efficiency map.

From equation (4) the required current $i_{2}$ is evaluated assuming constant DC bus voltage $V_{d c}$ (i.e. neglecting current fast dynamics associated to power conversions and the DC voltage dependency on battery SOC)

$$
i_{2}=\frac{\Pi_{e 2}}{V_{d c}}
$$

Since the power train layout reported in Figure 2 consist in flywheel and a small battery pack a strategy for charging/discharging the battery and the flywheel has to be adopted. This is done by adopting a weighting factor $\xi$ as follows. Calling $i_{b}$ the battery current and $i_{1}$ the flywheel current, the required current for traction motor $i_{2}$ is given by the following equation

$$
i_{2}=\xi i_{b}+(1-\xi) i_{1}
$$

where $\xi$ is the weighting factor that depends on the flywheel discharging strategy which relies on battery SOC (State of charge) as reported in figure $4 . \quad$ The shape of this function is chosen considering that the battery life is increases if the SOC remains as low as possible avoiding long period in full charg status [27]. Battery discharging is then preferred when the SOC is greater than $40 \%$ as given by $\xi$ factor.

The battery SOC is then computed by integrating the require battery current (current is positive if discharging the battery, negative if regenerative)

$$
S O C=S O C_{0}-\frac{1}{C} \int_{0}^{t} i_{b} d t
$$


where $C$ is the battery capacity. The flywheel mechanical power $\Pi_{m f}$ is instead computed from the required flywheel current $i_{1}$ and the efficiency of the flywheel motor $\left(\eta_{m 1}\right)$ and inverter $\left(\eta_{i 1}\right)$ :

$$
\Pi_{m f}=\frac{V_{d c} i_{1}}{\eta_{i 1} \eta_{m 1}}
$$

\section{Flywheel mass and geometry}

To evaluate the flywheel required energy, it is not enough to consider the previous model since the flywheel energy depends on flywheel mass that affects the total vehicle mass thus the required energy. Some assumptions on the flywheel geometry has to be considered in order to describe the flywheel with only one parameter, i.e. the flywheel mass.

An important parameter that defines the energy content of a flywheel is the specific energy, i.e. the amount of energy that can be stored for every kilogram of flywheel mass. This quantity must be maximized to minimize the flywheel mass. For a rotating object, the specific kinetic energy $e$ is expressed as

$$
e=\frac{E}{m}=\frac{1}{2} j \omega^{2}
$$

where $j$ is the specific moment of inertia $(j=J / m)$ and $\omega$ the spin speed. Considering a rim geometry and the mechanical properties of the flywheel material, as proposed in [16], the maximum achievable energy density is given by the maximum tensile stress and the material density:

$$
e=\frac{E}{m}=\frac{\sigma_{\theta}}{2 \rho}
$$

where $\sigma_{\theta}$ is the limit tangential stress and $\rho$ is the material density. Given the external and internal radius of the flywheel $\left(r_{\text {fly }}\right.$ and $r_{\text {internal }}$ respectively) there is a maximum rotational speed that fulfills equation (10) according to the material properties.

Five materials have been considered: kevlar, Toray T1000G composite, Toray T1000G fiber, Toray Torayca T800H and Carbon T1000. Details can be found in $[16,15,17,3,2]$. These materials can be defined as traditional. Other ones like carbon nanotubes maybe be used in future, even if they seem unrealistic today, as they promise a huge improvement in performances.

Table 2 reports the energy density of the five considered materials. 
Table 2: Materials energy density

\begin{tabular}{lr}
\hline Material & Energy density $[\mathrm{kWh} / \mathrm{kg}]$ \\
\hline \hline Kevlar & 0.1248 \\
Toray T1000G Composite & 0.235 \\
Toray T1000G Fiber & 0.491 \\
Toray Torayca T800H & 0.421 \\
Carbon T1000 & 0.1782 \\
\hline
\end{tabular}

The Toray T1000G Fiber [2] turns out to be the best performing material. According to the geometrical limits imposed by space onboard the vehicle, the maximum allowed speed for a flywheel made of this material spans from 62945 rpm to $169370 \mathrm{rpm}$.

However, it is difficult to find an electric motor that runs in this speed range delivering at the same time the requested torque. The motor is supposed to be directly connected to the flywheel shaft so to avoid the use of a gearbox which will dissipate some energy and, furthermore, it would be difficult to lubricate in vacuum. For this reason, the maximum flywheel angular speed $\omega_{\max }$ has been set to $30000 \mathrm{rpm}$, as a commercial motor with this performance exists.

This assumption is a limitation since the material is not exploiting all it strength potential as expressed by maximum specific energy of equation 10 .

Given this limitation and considering the available space onboard the car (external ring maximum radius $r_{f l y}=0.25 \mathrm{~m}$ ), the possibility to have a more power-requesting driving cycle and the overall system efficiency an iterative algorithm is used to size the flywheel.

At this point an algorithm has been implemented to determine the required flywheel mass that allows the car to accomplish the mission (see Figure 5).

The result of the optimization in term of flywheel geometry is:

- external ring radius $r_{f l y}=0.25 \mathrm{~m}$,

- internal ring radius $r_{i n t}=0.15 \mathrm{~m}$,

- ring height $h=0.15 \mathrm{~m}$,

- maximum angular speed $\omega_{\max }: 30000 \mathrm{rpm}$.

A couple of flywheels with that geometry fits inside the car; the positioning on board the vehicle is shown in figure 6 .

To verify that the obtained solution is the only one feasible for the problem, the selected flywheel shape and dimensions are fixed while the material is changed. For this reason, a comparison between the UTS of different materials and the actual value for this geometry has been made [16]. In Table 3 results are shown.

It can be seen that common materials like aluminum and steel are not able to resist to the stress induced by centrifugal force. As for the Advantex E-glass, the safety factor is just 1.56 and the mass is $6 \mathrm{~kg}$ higher than carbon fiber materials, this is not a convenient solution by a pure technical point of view. The only 


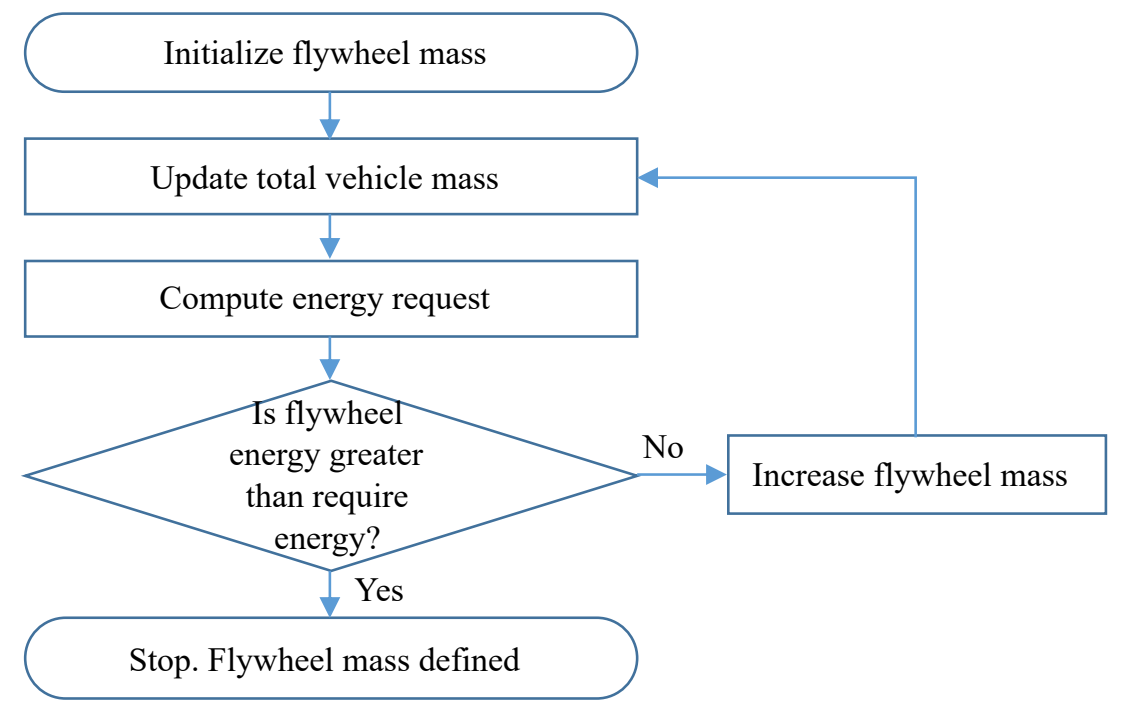

Figure 5: Iterative algorithm used to define the flywheel mass.

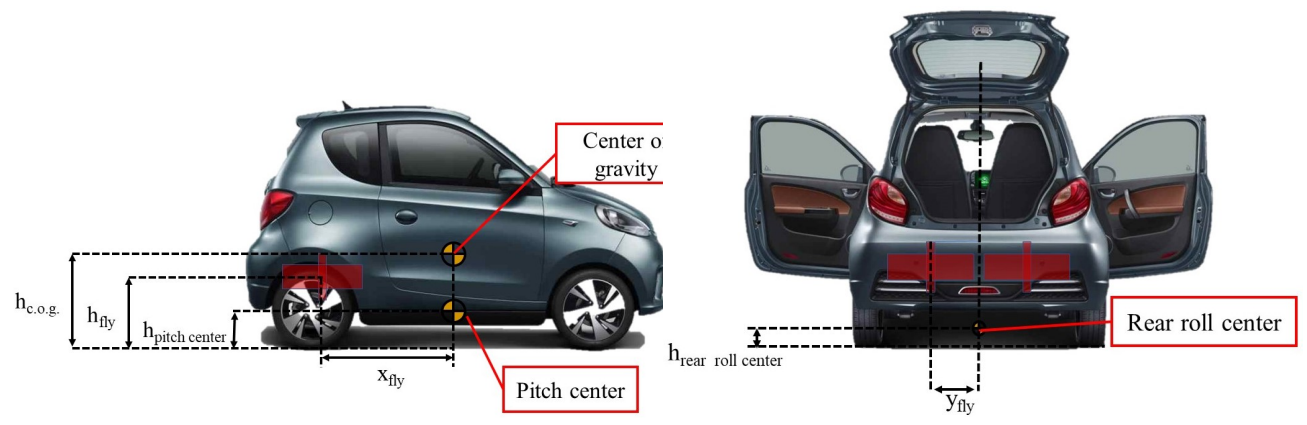

Figure 6: Flywheels position on board the vehicle 
Table 3: Materials comparison

\begin{tabular}{|c|c|c|c|c|c|c|}
\hline Material & $\begin{array}{l}\text { Density } \\
{\left[\mathrm{kg} / \mathrm{m}^{3}\right]}\end{array}$ & $\begin{array}{l}\mathrm{UTS} \\
{[M P a]}\end{array}$ & $\begin{array}{l}\text { Mass } \\
{[k g]}\end{array}$ & $\begin{array}{l}\text { Energy } \\
{[k W h]}\end{array}$ & $\begin{array}{l}\text { Actual } \\
\text { stress } \\
{[M P a]} \\
\end{array}$ & $\begin{array}{l}\text { Safety } \\
\text { factor } \\
]\end{array}$ \\
\hline $\begin{array}{l}\text { Aluminum } \\
7075\end{array}$ & 2810 & 572 & 53 & 3.09 & 1179 & 0.49 \\
\hline 17-7 $\quad \mathrm{PH}$ & & & & & & \\
\hline $\begin{array}{l}\text { Stainless } \\
\text { steel }\end{array}$ & 7800 & 1650 & 148 & 8.62 & 3293 & 0.50 \\
\hline $\begin{array}{l}\text { Titanium } \\
\text { Ti-15V- } \\
3 \mathrm{Cr}-3 \mathrm{Al}- \\
3 \mathrm{Sn}\end{array}$ & 4760 & 1380 & 89.7 & 5.23 & 1997 & 0.69 \\
\hline $\begin{array}{l}\text { Advantex } \\
\text { E-glass } \\
\text { Toray }\end{array}$ & 2146 & 1400 & 40.5 & 2.36 & 900 & 1.56 \\
\hline $\begin{array}{l}\text { T1000G } \\
\text { composite } \\
\text { Toray }\end{array}$ & 1800 & 3040 & 33.9 & 1.98 & 755 & 4.03 \\
\hline $\begin{array}{l}\text { T1000G } \\
\text { fiber }\end{array}$ & 1800 & 6370 & 33.9 & 1.98 & 755 & 8.44 \\
\hline kevlar & 1380 & 1240 & 26 & 1.52 & 579 & 2,14 \\
\hline $\begin{array}{l}\text { Toray } \\
\text { T800H }\end{array}$ & 1810 & 5490 & 34.1 & 1,99 & 759 & 7.23 \\
\hline $\begin{array}{l}\text { Carbon } \\
\text { T1000 }\end{array}$ & 1520 & 1950 & 28.7 & 1.67 & 638 & 3.06 \\
\hline
\end{tabular}



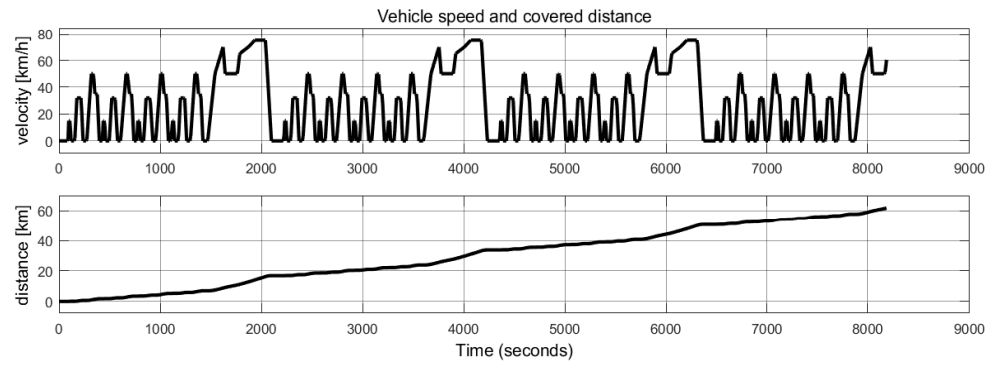

Figure 7: Vehicle velocity and distance

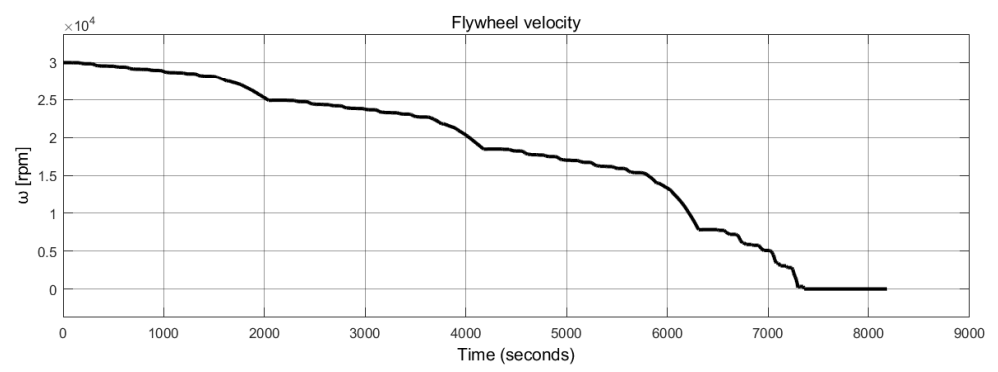

Figure 8: Flywheel velocity

other material which can resist is kevlar, but its low energy does not guarantee the target driving-range. In conclusion, Toray T1000G fiber is not the only choice, but a high performance material is necessary. The chosen configuration implies a mass of $33.9 \mathrm{~kg}$ per each flywheel, which guarantees a driving-range of $61.7 \mathrm{~km}$ considering also the energy stored in the battery until it reaches the $30 \%$ of SOC. In figure 7 the covered distance and the vehicle velocity are shown, while in figure 8 the velocity profile of the flywheel is visible. The fact that the vehicle continues to run even if the flywheel velocity is zero is due to the recovered energy into the battery during the previous deceleration; the flywheel energy is finished and the final part of the trip is performed thanks to the residual battery energy.

\section{Effects on vehicle handling: the gyroscopic ef- fects}

Once a feasible solution has been found in terms of size and stored energy, it is necessary to evaluate the possible side-effects produced by a such high speed rotating object. In particular, this section studies the gyroscopic effects produced by the flywheel and their influence on vehicle dynamics.

As previously stated, an even number of flywheels with opposite spin is required, and due to room space limitations, only two flywheels can be installed 
on board the ZD-1. The issue is that, as the vehicle is a dynamic system with three rotational degrees of freedom, gyroscopic effects induced by the high rotational speed of each flywheel are present. This phenomenon, for a body rotating in space, is described by Euler's equations:

$$
\begin{aligned}
& J_{x} \dot{\omega}_{x}+\left(J_{z}-J_{y}\right) \omega_{y} \omega_{z}=M_{x} \\
& J_{y} \dot{\omega}_{y}+\left(J_{x}-J_{z}\right) \omega_{z} \omega_{x}=M_{y} \\
& J_{z} \dot{\omega}_{z}+\left(J_{y}-J_{x}\right) \omega_{x} \omega_{y}=M_{z}
\end{aligned}
$$

where, adopting the subscript $i$ for $x, y$ or $z, M_{i}$ is the component of the applied external torques, $J_{i}$ is the flywheel principal moments of inertia and $\omega_{i}$ the components of the angular velocity about the corresponding principal axis $(i)$. In the case of flywheel supported on the vehicle chassis in still conditions, the flywheel $z$ axis is aligned with the vehicle yaw axis, while $x$ and $y$ correspond to roll and pitch axes respectively.

From these equations the gyroscopic torques $M_{i, G}$ are:

$$
\left\{\begin{array}{l}
M_{x, G}=\left(J_{y}-J_{z}\right) \omega_{z} \omega_{y} \\
M_{y, G}=\left(J_{z}-J_{x}\right) \omega_{z} \omega_{x} \\
M_{z, G}=\left(J_{x}-J_{y}\right) \omega_{x} \omega_{y}
\end{array}\right.
$$

This equation well explains why attention must be put on this topic since in this application the flywheel rotating speed $\Omega$ reaches $30000 \mathrm{rpm}$. A rotation of the flywheel in a direction which is perpendicular to the spinning axis produces a gyroscopic torque as described in eq (14). This means that a pitch rotation of the car generates a torque in roll axis direction and viceversa.

In figure 9 the direction of the spinning rotations is reported along with the reference system of the vehicle.

If the two flywheels are spinning at the same angular speed $\Omega$ about rotating axis the gyroscopic moments are the same for the two flywheel. The resulting gyroscopic moment is the sum of the ones produces by each single flywheel. If instead the flywheels are rotating in opposite direction with same magnitude, the gyroscopic torques cancels each other. I.e., by the point of view of the chassis, the two effects delete each other, producing no unbalance.

Keeping in mind that the gyroscopic torque must be seen by the fixed reference system, during a turn the total angular speed of the flywheel with respect to the ground is the algebraic sum between the yaw rate of the vehicle $(\dot{\psi})$, and the spinning velocity of the flywheel with respect to the chassis $\Omega$. Supposing that the car has a positive yaw rate $(\dot{\psi}>0)$ the absolute angular speed $\Omega$ in $z$ direction of the left $l$ and right $r$ flywheels are

$$
\omega_{z, r}=\Omega+\dot{\psi} \quad \omega_{z, l}=-\Omega+\dot{\psi}
$$

which means that the two flywheels are not perfectly balanced by the point of view of gyro effect due to the vehicle yaw rate. The angular speed unbalance is in fact equal to $2 \dot{\psi}$. 


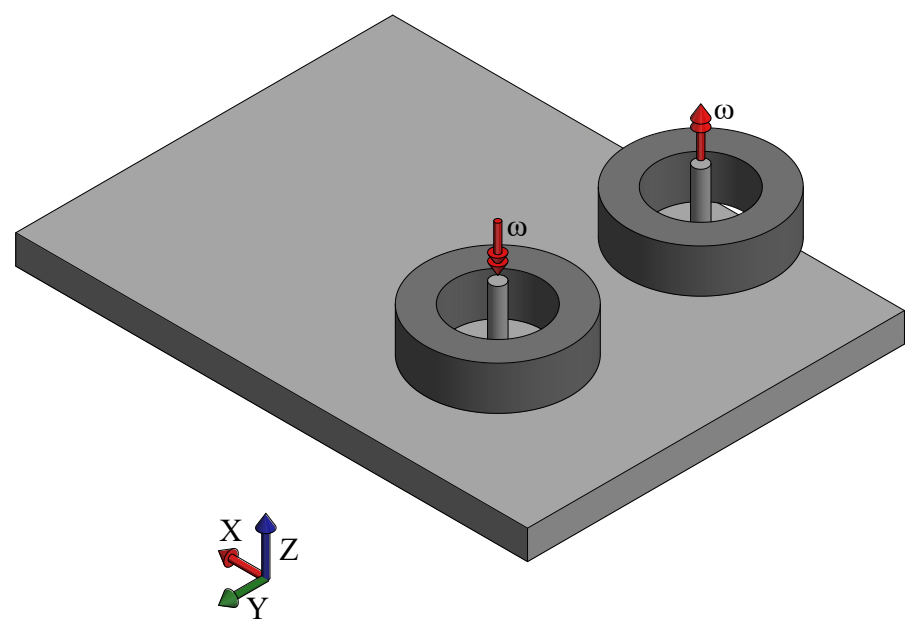

Figure 9: Flywheel orientation and spinning rotation with respect to vehicle chassis

Table 4: Gyroscopic moments induced while turning and braking or acceleration

\begin{tabular}{lcc} 
& $\begin{array}{c}\text { turn left } \\
(\dot{\psi}>0)\end{array}$ & $\begin{array}{c}\text { turn right } \\
(\dot{\psi}<0)\end{array}$ \\
\hline$M_{x, G}(\dot{\theta}<0)$ & $>0$ & $<0$ \\
accel $(\dot{\theta}>0)$ & $<0$ & $>0$ \\
brake $(\dot{\theta}>0$
\end{tabular}

This unbalance produces pitch and roll moment on the chassis, as better described in the following, but these effects are much smaller than the effect generated when adopting one single flywheel.

The unbalance produces, in fact, a coupling effect between the pitch and roll motions of the chassis, while pitch and roll are not affecting the flywheel spinning speed because of its cylindrical symmetry $\left(J_{x}=J_{y}\right)$ that makes the $M_{z, G}$ equal to zero.

As summarized in table 4 , when the car has a positive pitch motion $(\dot{\theta}>0)$, i.e. vehicle brakes, while turning left (yaw rate $\dot{\psi}>0$ ), the gyroscopic moment in roll direction is negative, this will reduce the roll angle that is positive due to centrifugal inertia force. On the other hand, if vehicle accelerates during the same turn $(\dot{\theta}<0), M_{x, G}$ is positive thus increases the roll angle.

As for roll instead, when entering in a left turn, both yaw rate $\dot{\psi}$ and roll rate $\dot{\phi}$ are positive. This produces a negative pitch torque which makes the front of the vehicle to rise; in a right turns the same happens, since both the yaw velocity and the roll rate have changed their directions. 

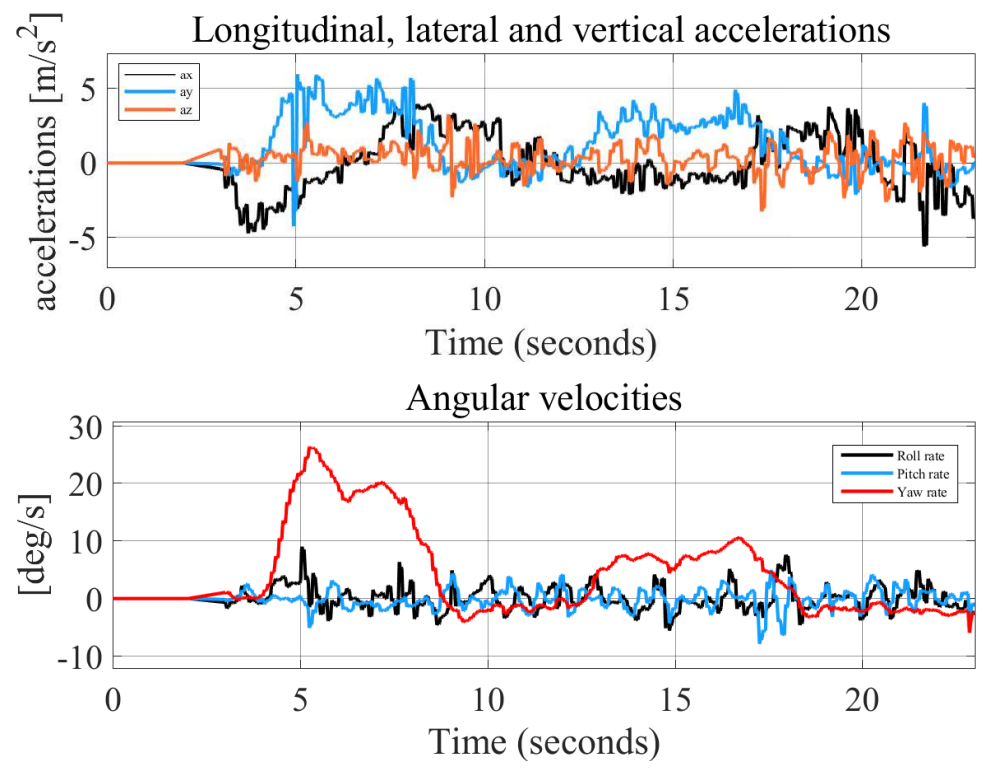

Figure 10: Time history of the chassis accelerations and angular velocity components measured in a normal driving maneuver.

To understand the trend of these torques, simulations have been performed relying on Multibody Simscape model. These simulations have two main targets:

- evaluate the gyroscopic effects on vehicle dynamics by computing the resulting torques exerted by the flywheel case on the vehicle chassis;

- evaluate the single flywheel gyroscopic torques necessary to dimension the flywheel bearings as better described in the following section.

The flywheel case is a rigid box that hosts the flywheels to which they are connected by means of pin joints pointing in vertical direction with imposed rotating speed $\Omega$ and $-\Omega$ respectively for right and left flywheel. The flywheels case is then linked to the vehicle chassis by means of engine rubber mounts, with given stiffness and damping coefficients in $x, y$ and $z$ direction. Chassis motion is then imposed applying the time history of the chassis angular velocity (yaw, pitch and roll). As an example, Figure 10 reports the time histories the chassis accelerations and angular velocity components measured in a normal driving maneuver. Those measurements have been fed to the multibody model to reproduce a real excitation of the car. The model outputs are then the gyroscopic torques exerted by the flywheel case to the chassis. As shown in Figure 11, even if the flywheel rotating speed is $30000 \mathrm{rpm}$, remembering equation (14), the resulting gyroscopic torques are small because of the small values of chassis angular velocity components.

As it can be noticed there is no yaw effect: it is a great advantage as the 

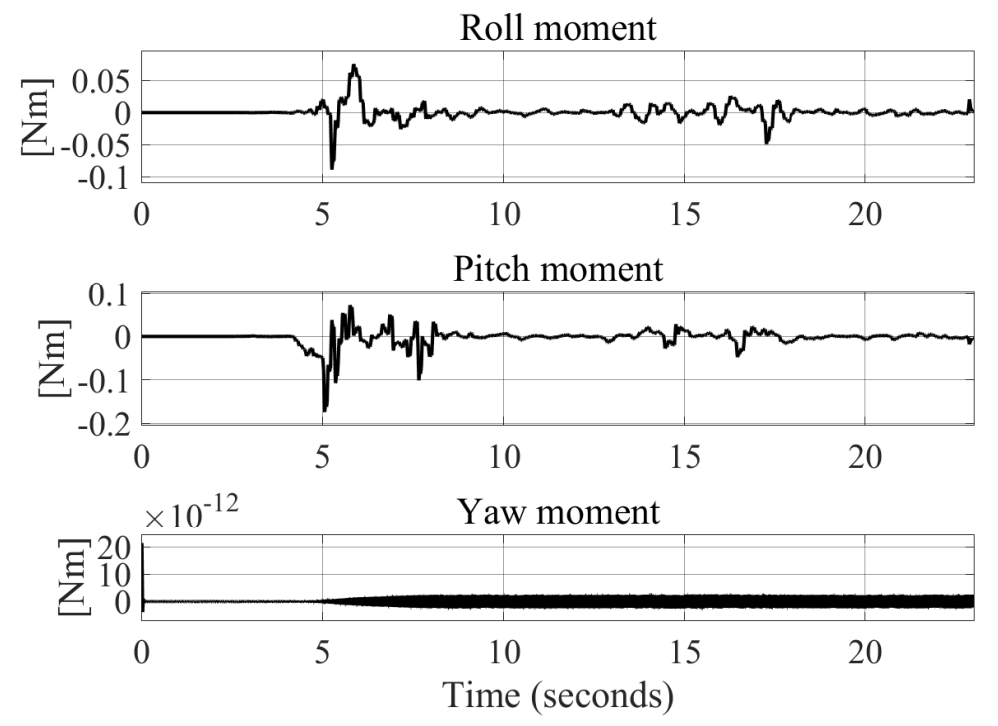

Figure 11: Final test gyroscopic torques

vehicle does not try to modify the imposed trajectory by the driver. As for unbalances on the other axis, they are certainly present specially when the yaw rate is high (since the gyroscopic torque modulus depends on it) but their amplitude remains orders of magnitude lower than other effects. Moreover, it can be noted that both pitch and roll unbalancing have the same order of magnitude, this is because both rates and lever arms have the comparable magnitude among themselves.

From previously presented simulation, it is also possible to obtain the forces required to the flywheel bearings. By considering the flywheel to be connected to the case by means of two bearings, one in bottom and one in the upper part of the flywheel, the gyroscopic moments and the flywheel inertia forces are then balanced by couples of forces in x-y plane as schematized in Figure 12.

In table 5 forces at the bearing in $\mathrm{x}-\mathrm{y}$ plane are expressed in terms of peak value, mean and RMS.

Table 5: Forces at the bearings

\begin{tabular}{lrrrrrr}
\hline & \multicolumn{2}{c}{ Peak $[N]$} & \multicolumn{2}{c}{ Mean $[N]$} & \multicolumn{2}{c}{ RMS $[N]$} \\
& left & right & left & right & left & right \\
\hline \hline$F_{x}$ upper & 2478 & 2387 & 25 & -45 & 558 & 546 \\
$F_{y}$ upper & 2153 & 2161 & 25 & -17 & 531 & 526 \\
$F_{x}$ lower & 2397 & 2489 & -44 & 25 & 547 & 559 \\
$F_{y}$ lower & 2161 & 2152 & -20 & 27 & 527 & 531 \\
\hline
\end{tabular}




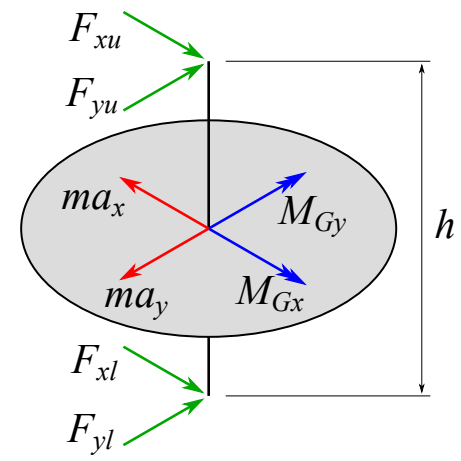

Figure 12: Scheme for the computation of bearing radial forces.

\section{Bearings}

Once the general dimension of the flywheel has been defined and the gyroscopic moments evaluated, the design focuses on the bearings.

Two main problems must be faced when designing the flywheel bearing: the system stability and the power losses induced by bearings. The first one has to be granted, this means that unstable behavior has to be avoided, e.g. instability may arise in case of hydraulic bearings. Differently from static flywheels used in energy storage for still applications, mounting the flywheel on a car means that the flywheel is subject to accelerations as shown in the previously presented simulation. The inertia force and gyroscopic moment of the flywheel are absorbed by bearings that have to damp the flywheel oscillations. A way to reduce vibrations coming both from the road unevenness and from the vehicle dynamics can be the gimbal [21] [23]. But the poor capability to manage gyroscopic effect and the increase of complexity have brought to the discarding of this solution.

The second effect, i.e. the energy dissipation, must be limited as much as possible to increase the available energy for the motion of the vehicle and avoid the complete stop of the flywheel when the vehicle is not moving. As batteries show power drain when not used, flywheel losses energy because of friction. Having removed the air friction thanks to vacuum environment, bearing friction can still be present.

Once these considerations are made, there are several bearing types that can be considered:

- hydrodynamic bearings;

- steel ball bearings;

- passive magnetic bearings (PMB);

- active magnetic bearings (AMB);

- ceramic ball bearings (CBB). 


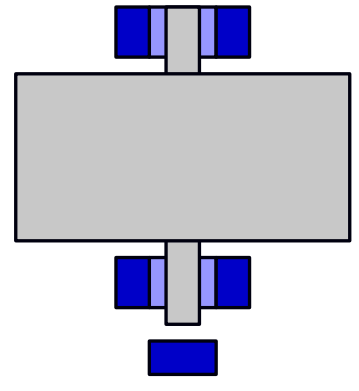

(a)

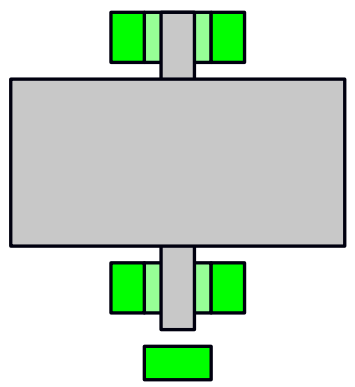

(c)

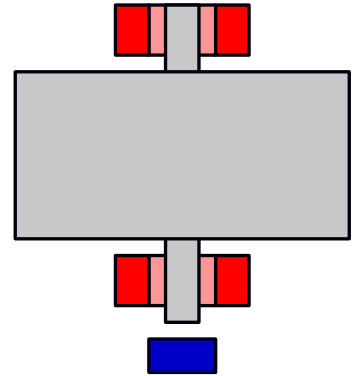

(b)

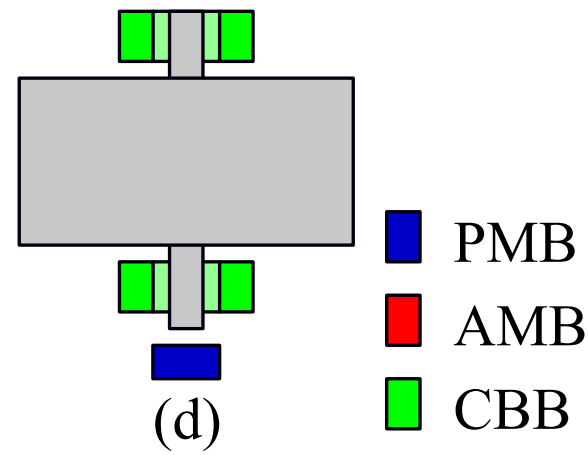

Figure 13: simulated bearings configurations.

Hydrodynamic bearings are immediately discarded due to the infeasibility in vacuum environment. Steel ball bearings are also discarded due to speed limitation and high friction losses.

Passive and active magnetic bearing seems a promising solution that allows in fact to complete cancel the power dissipation due to friction. This is true for passive but not for active that use energy to generate the magnetic field. Ceramic ball bearing place in between, they are better than steel ball bearing but they dissipate energy due to friction.

To choose the best solution several configurations have been simulated. In the simulations, the forces acting on the bearing are the one taken from previously presented simulation that account for on-board measured acceleration and angular speeds. The considered configurations are reported in figure 13. In each configuration two radial bearing are connected to the top and bottom end of the flywheel shaft while one axial bearing supports the flywheel weight in the bottom part. 


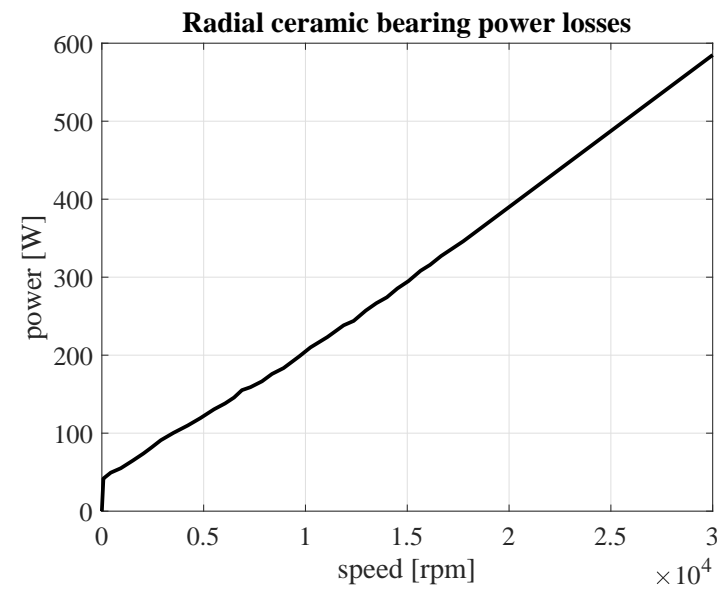

Figure 14: Power dissipation of $\mathrm{CBB}$ as function of rotating speed.

\subsection{Bearings modeling}

To evaluate different bearing configurations, each bearing type is modeled to simulated the behavior of the flywheel in the previously presented dynamic conditions, i.e. in a normal driving scenario with acceleration induced on vehicle chassis due to vehicle motion and road irregularity.

PMB are modeled accounting for their force-distance characteristics as shown in $[11,12,10]$. The problem of PMB is the lack of damping which makes them unsuitable for the target application. The vibrations induced by the chassis motion result in a dynamic instability of the flywheel.

AMB are modeled according to [8] where a PID controller is used to generate a bearing force that maintains the shaft centered with respect to the bearing. By simulating the bearing with that model it is possible to evaluate the bearing energy consumption which is of $0.213 \mathrm{kWh}$. Comparing this energy request with the total flywheel energy, the average AMB efficiency is about $90 \%$ which is considered too low for the application.

CBB are instead modeled accounting for their radial and axial stiffness and accounting for the power dissipation characteristic as function of rotating speed as reported in Figure 14 (data taken from [1]).

Several considerations can be drawn from the analyzed configurations:

PMBs suffer from two main issues: firstly the stiffness produced by the PMBs taking into account the application limited space is too small for the calculated forces. Secondly, due to Earnshaw's theorem, stability cannot be achieved due the absence of damping.

AMBs are stabilizing the system but their main drawback is the power consumption which can considerably affect the overall driving-range. To reduce the energy consumption gimbal is necessary to reduce bearings forces. 
With the gimbal, in fact, the energy required by $\mathrm{AMB}$ reduce the car driving-range from $61.7 \mathrm{~km}$ to $50 \mathrm{~km}$.

CBBs represents a good compromise. They guarantee stability but only small forces can be applied to limit the energy dissipation which strictly depends on applied forces.

Referring to figure 13, the following considerations can be done regarding each analyzed solution:

a) is unfeasible due to stability issue associated to the use of only PMB;

b) is unfeasible due to high power consumption necessary to control the flywheel position;

c) is unfeasible due to high power losses. With this configuration the drivingrange lowers by $33 \%$ with respect to simulation without bearings. The most detrimental drawback is that the dissipation of all the stored energy happens in five hours;

d) is found to be the best compromise solution since using a PMB axially to sustain the weight and two radial ceramic bearings to prevent lateral and longitudinal motions, the forces and thus the dissipation in ceramic bearing are reduced. During the simulated driving cycle the energy dissipated is found to be $0.5 \%$ of the total energy stored in the flywheel.

Once the bearings configurations is chosen, the mounting of the flywheel case is given. The case is linked to the vehicle chassis by means of engine mounts, that allow the case to have independent degrees of freedom with respect to the chassis, moreover the bushings filter the acceleration transmitted from chassis to flywheel. In figure 15 a visual layout of the complete system is shown. The yellow cylinders are the motor stators which are directly coupled to the flywheels without any reduction gear due to lubrication problems in vacuum environment. Ceramic radial bearings and PMBs to compensate the static weight are also present. The four springs in parallel to the dampers represent the case supports. Figure 16 and figure 17 reports instead the simulation of the driving cycle accounting also for the bearings. Figure 16 reports the power output from the flywheel and the power requested by the wheel to drive the car. Negative power values mean regenerative braking. The required power when the car is still accounts for the bearings power request. Figure 17 reports instead the total energy delivered by main components of the powertrain layout. As it can be noticed, the bearing consumption is the most relevant term and the total powertrain efficiency, total car required energy over total flywheel energy, is $41 \%$.

\section{Conclusions}

This paper studied the possibility of adopting a flywheel to power a city car. Firstly an energetic model of the car has been developed to evaluate the energy 


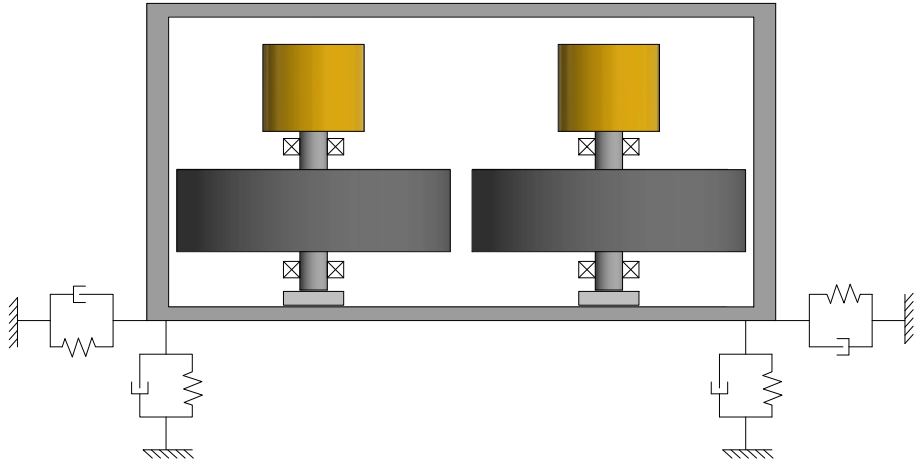

Figure 15: System visual layout

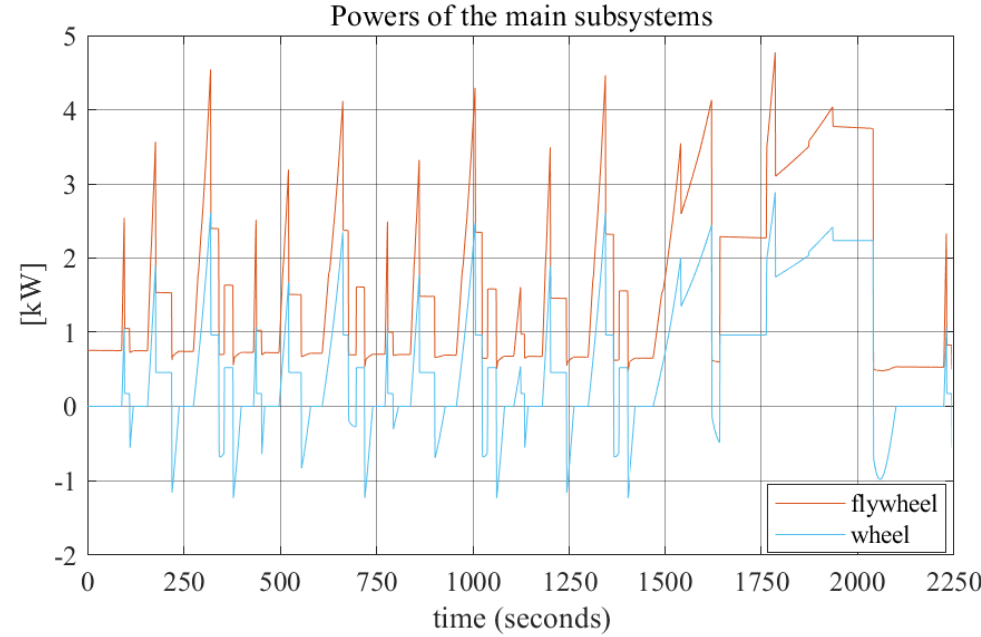

Figure 16: Power consumption for 1 loop of the driving cycle. 


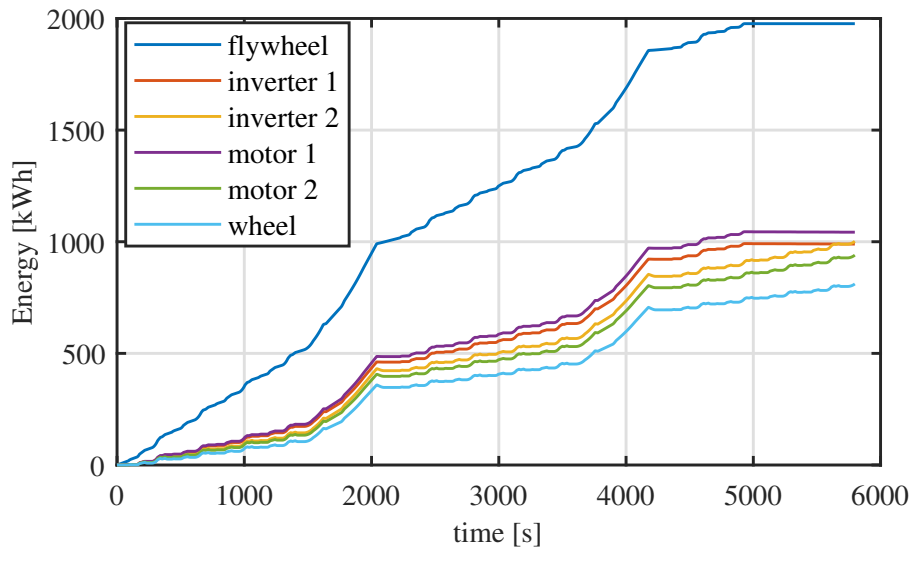

Figure 17: Energy consumption for main subsystems for simulated driving cycle.

required to drive the car in a typical trip. An iterative process has then been adopter to correctly dimension the flywheel according to a simplified geometry and accounting for available room onboard the car. The flywheel dimension and inertia are accounted as function of the chosen material in terms of mass density and mechanical limit stress. Once the flywheel dimensions and mass has been defined, the gyroscopic effect induced by the flywheel on car dynamics have been studied by means of multibody simulation. Results show that gyroscopic effect are negligible if a even number of flywheels is considered and flywheels are two by two spinning in opposite direction and with same speed. Finally, the bearings type and configuration has been analyzed. The most performing solution resulted to be an hybrid solution that uses ceramic ball bearings to support radial forces while one passive magnetic bearing sustains the flywheel weight.

\section{References}

[1] Ceramics bearing for extreme special environments. Technical report, Jtekt Corporation.

[2] ToraycaT1000G Data Sheet.

[3] ToraycaT800H Data Sheet.

[4] The new type vehicle with electromagnet flywheel for electric drive $\mathcal{E}$ energy recuperation, 2014.

[5] M.L. Bacci, F. Cheli, E. Sabbioni, D. Tarsitano, and M. Vignati. Aging models for high capacity LiFePO $<$ inf $>4</$ inf $>$ cells. In 2017 International Conference of Electrical and Electronic Technologies for Automotive, 2017. 
[6] Michael A. Conteh and Emmanuel C. Nsofor. Composite flywheel material design for high-speed energy storage. Journal of Applied Research and Technology, 14(3):184-190, jun 2016.

[7] Xingjian Dai, Kai Zhang, and Xiao-zhang Zhang. Design and test of a 300Wh composites flywheel energy storage prototype with active magnetic bearings. Renewable Energy and Power Quality Journal, 1(9):380-385, may 2011.

[8] Luiz de Paula do Nascimento and Carlos Henrique de Oliveira Arantes. Control parameters of active magnetic bearings supporting rotating systems. International Journal of Latest Research in Science and Technology, 2014 .

[9] A. Dhand and K. Pullen. Review of flywheel based internal combustion engine hybrid vehicles. International Journal of Automotive Technology, 2013.

[10] Krzysztof Falkowski. Simulation of passive magnetic bearing using comsol multiphysics. In COMSOL Conference in Stuttgrat.

[11] Alexei V. Filatov and Eric H.Maslen. Passive magnetic bearing for flywheel energy storage system. IEEE transactions on magnetics, 2001.

[12] Guillaume Filion, Jean Ruel, and Maxime R. Dubois. Reduced-friction passive magnetic bearing: Innovative design and novel characterization technique. Machines, 2013.

[13] S.M. Mousavi G, Faramarz Faraji, Abbas Majazi, and Kamal Al-Haddad. A comprehensive review of flywheel energy storage system technology. Renewable and Sustainable Energy Reviews, 2016.

[14] Shiva Habibi, Frances Sprei, C Englundn, Stefan Pettersson, Alexey Voronov, Johan Wedlin, and Henrik Engdahl. Comparison of free-floating car sharing services in cities. In European Council of Energy Efficient Economy (ECEEE) Summer Study, Presqu'̂̀le de Giens, France, 29 May3 June, 2017, 2017.

[15] Stefan Hartl, Alexander Schulz, Harald Sima, Thomas Koch, and Manfred Kaltenbacher. A static burst test for composite flywheel rotors. Appl Compos Mater, 2016.

[16] Magnus Hedlund, Johan Lundin, Juan de Santiago, Johan Abrahamsson, and Hans Bernhoff. Flywheel energy storage for automotive applications. Energies, 2015.

[17] Khaled Itani, Alexandre De Bernardinis, Zoubir Khatir, and Ahmad Jammal. Comparative analysis of two hybrid energy storage systems used in a two front wheel driven electric vehicle during extreme start-up and regenerative braking operations. Energy Conversion and Management, 2017. 
[18] Hongliang Li, Jiangwei Chu, Jialu Li, Pengfei Cui, and Zhanzhong Wang. Energy recovery data characteristic extraction of flywheel energy storage control system for vehicular applications. Advances in Mechanical Engineering, 2017.

[19] Teng Liu, Xiaolin Tang, Hong Wang, Huilong Yu, and Xiaosong Hu. Adaptive Hierarchical Energy Management Design for a Plug-In Hybrid Electric Vehicle. IEEE Transactions on Vehicular Technology, 68(12):11513-11522, dec 2019.

[20] Keith R. Pullen Mustafa E. Amiryar. A review of flywheel energy storage system tecnologies and their applications. Technical report, School of Mathematics, Computer Science and Engineering, University of London, 2017.

[21] R. Peña-Alzola, R. Sebastián, J. Quesada, and A. Colmenar. Review of flywheel based energy storage systems. In International Conference on Power Engineering, Energy and Electrical Drives.

[22] Yechen Qin, Xiaolin Tang, Tong Jia, Ziwen Duan, Jieming Zhang, Yinong $\mathrm{Li}$, and Ling Zheng. Noise and vibration suppression in hybrid electric vehicles: State of the art and challenges. Renewable and Sustainable Energy Reviews, 124(October 2019):109782, may 2020.

[23] Budi Rachmanto, Kenzo Nonami, Kenta Kuriyama, Hiroshi Shimazaki, Takahiro Kagamiishi, and Tomoki Moriya. A study on amb flywheel powered electric vehicle. Journal of System Design and Dynamics, 2009.

[24] Maciej Różewicz. Shape optimization of a flywheel. $A u$ tomatyka/Automatics, 18(1):23, 2014.

[25] Igor Trivić. Comparative Analysis of Alternative Hybrid System for Automotive Applications. PhD thesis, Alma Mater Studiorum - University of Bologna, 2012.

[26] Michele Vignati, Davide Tarsitano, Mattia Bersani, and Federico Cheli. Autonomous Steer Actuation for an urban Quadricycle. In 2018 International Conference of Electrical and Electronic Technologies for Automotive, pages 1-5. IEEE, jul 2018.

[27] Evelina Wikner and Torbjörn Thiringer. Extending Battery Lifetime by Avoiding High SOC. Applied Sciences, 8(10):1825, oct 2018.

[28] Çağlar İmer, Samet Kartal, and Mehmet Onur Genç. Stress Behavior Improvement Analysis of Automobile Flywheel Experimentally and $\mathrm{Nu}-$ merically. International Journal of Automotive Science And Technology, 2(1):1-6, 2018. 